\title{
A population-based observational study comparing Cervista and Hybrid Capture 2 methods: improved relative specificity of the Cervista assay by increasing its cut-off
}

Gerd Boehmer ${ }^{1}$, Lisa Wang ${ }^{2}$, Angelika Iftner ${ }^{3}$, Barbara Holz ${ }^{3}$, Juliane Haedicke ${ }^{3}$, Reinhard von Wasielewski ${ }^{1}$, Peter Martus ${ }^{2}$ and Thomas Iftner ${ }^{3^{*}}$

\begin{abstract}
Background: High-risk human papillomavirus (HR HPV) testing has been shown to be a valuable tool in cervical cancer screening for the detection of cervical pre-cancer and cancer.

Methods: We report a purely observational study evaluating HR HPV prevalences in residual liquid-based cytology (LBC) samples using both the Cervista HPV HR Test and the Digene Hybrid Capture 2 High-Risk HPV DNA Test (HC2) in a sample of 1,741 women aged $\geq 30$ years of a German routine screening population of 13,372 women. Test characteristics were calculated and a novel method for measuring test performances was applied by calculating ratios of sensitivity or specificity.

Results: The overall agreement of both tests for detection of HR HPV was excellent $(k=0.8)$. Relative sensitivities for the detection of histologically confirmed severe cervical intraepithelial dysplasia (CIN3+) were similar for both HPV-tests, which was confirmed by the ratio analysis. However, discrepancy analysis between the Cervista HPV HR test and HC2 revealed a high false positive rate of the Cervista HPV HR test in the cytology normal category.

Conclusions: Performance of the Cervista HPV test in cervical specimens with abnormal cytology is comparable to $\mathrm{HC2}$ as both tests were highly sensitive and specific for the detection of high grade cervical disease. We also demonstrate evidence that modification of the cut-off values drastically reduces the false positive rate in the cytology normal category without affecting the detection of CIN3+, which ultimately improved specificity of the Cervista HPV HR assay.
\end{abstract}

Keywords: Cervista, Hybrid capture, HPV, Cervical cancer screening

\section{Background}

In Germany, the cervical cancer mortality rate has notably decreased since the introduction of gynaecological screening for cervical cancer in 1971 [1]. Annual opportunistic screening is usually performed by conventional cytology (Pap-smear) and is covered by health insurances for women aged 20 years or older. Nevertheless, 4,900 new cases and approximately 1,600 deaths of cervical cancer

\footnotetext{
* Correspondence: Thomas.iftner@med.uni-tuebingen.de

Equal contributors

${ }^{3}$ Division of Experimental Virology, Institute of Medical Virology, University Hospital Tbingen, Elfriede-Aulhorn-Str. 6, 72076 Tbingen, Germany Full list of author information is available at the end of the article
}

are observed each year [2] and 150,000 cases of cervical cancer precursors (CIN3) are diagnosed [3]. Cervical cancer accounts for $1.6 \%$ of all cancer deaths among women in Germany [2].

Persistent infection with high-risk human papillomaviruses (HR HPV) has been shown to be necessary for the development of cervical precancerous lesions and cancer [4]. Notably, the HR HPV types 16, 18, 31, 33, 35, 39, 45, 51, 52, 56, 58 and 59 have been defined as class I carcinogens and HPV type 68 as class IIa carcinogens by the IARC [5]. The majority of all cervical cancer cases are associated with the HR HPV types 16 and 18 [6]. The fact that HR HPV is the causative infectious agent of cervical cancer has led to

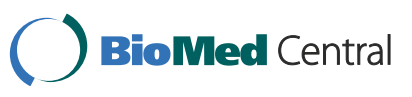

2014 Boehmer et al.; licensee BioMed Central. This is an Open Access article distributed under the terms of the Creative Commons Attribution License (http://creativecommons.org/licenses/by/4.0), which permits unrestricted use, distribution, and reproduction in any medium, provided the original work is properly credited. The Creative Commons Public Domain Dedication waiver (http://creativecommons.org/publicdomain/zero/1.0/) applies to the data made available in this article unless otherwise stated. 
the development and investigation of various HPV detection methods; and testing for HR HPV in addition to cytology is nowadays applied in cervical cancer screening [7-9]. Three DNA-based tests for the detection of the HR HPV group and one RNA-based assays for HPV detection have been approved by the US Food and Drug Administration (FDA) for routine cervical cancer screening (http://www. fda.gov/MedicalDevices/ProductsandMedicalProcedures/ InVitroDiagnostics/ucm330711.htm). Among these are the Digene Hybrid Capture 2 High-Risk HPV DNA test (HC2; QIAGEN, Hilden, Germany), the Cervista HPV HR test (CER; Hologic, Madison, WI), the cobas HPV Test (Roche, Pleasanton, USA) and the RNA-based APTIMA HPV Assay (Hologic, San Diego, CA). The cobas HPV Test has recently been approved for primary screening by the FDA (www.fda.gov).

The HC2 test for the collective detection of at least 13 carcinogenic HPV types $(16,18,31,33,35,39,45,51,52$, $56,58,59,68)[10]$ is a nucleic acid hybridisation assay with signal amplification using microplate chemiluminescence for semi-quantitative detection of HPV-DNA in cervical specimens. In addition to the 13 carcinogenic HPV types detected by $\mathrm{HC} 2$, the Cervista HPV HR assay also detects putative HR HPV type 66 [11]. The test principle employs the Invader chemistry, which is a signal amplification method recognizing specific nucleic acid sequences.

Performance comparisons of HR HC2 and the Cervista HPV HR test have previously been performed [12-22]. However, only a limited number of studies present data from both assays on the same residual LBC sample in comparison to cytology and histology results $[12,16,17,19]$. The objective of this study was therefore to evaluate the Cervista HPV HR assay in comparison to $\mathrm{HC} 2$ regarding relative sensitivity and specificity for the detection of high grade CIN3+ in women of a German routine screening population aged $\geq 30$ years using cervical samples collected in PreservCyt LBC medium. PreservCyt LBC cervical samples from 1,741 women with abnormal $(n=468)$, ASC-US $(n=20)$ as well as a random sample of women with normal cytology $(n=1,208)$ were analyzed by the Cervista HPV HR test and HC2.

\section{Methods}

\section{Study design}

The study was conducted in a routine screening population of 13,372 women $\geq 30$ years of age living in the Hannover area of Germany (Figure 1). Cervical samples were collected in PreservCyt Pap Test specimen collection medium (Hologic, Marlborough, MA) between February

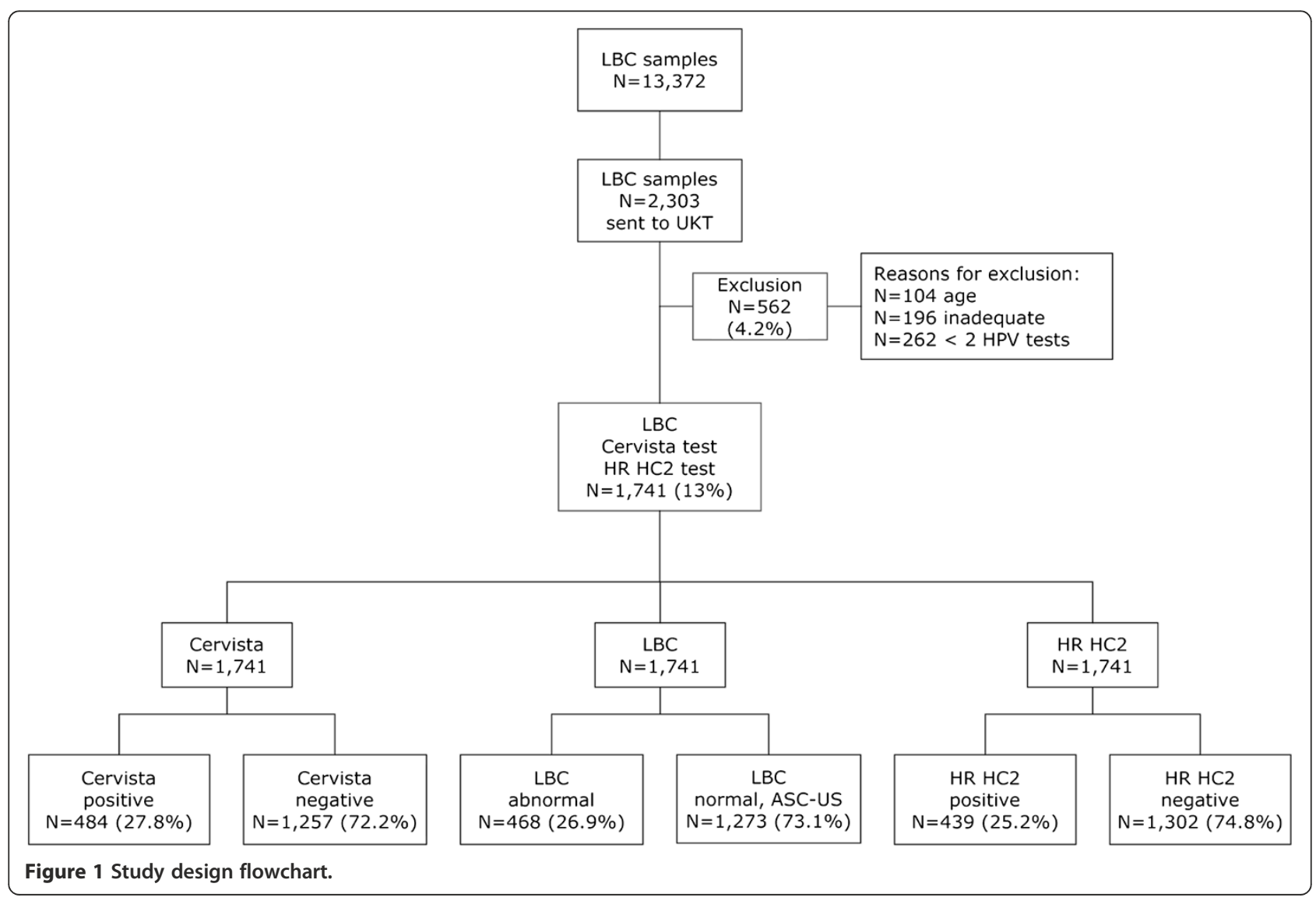


and June 2011 and cytology was tested within one week after collection in a central services laboratory (Amedes, Bad Mnder, Germany). As determined by the ethics committee (Ethikkommission bei der Deutschen rztekammer Niedersachsen), no ethical approval was required for this purely observational study, because residual patient samples were used that were completely anonymized and study results had no influence on the patients follow-up strategy. A total of 2,303 liquid based cytology (LBC) smears including all residual samples with abnormal and ASC-US cytology results as well as $10 \%$ of randomly selected normal LBC samples were sent to Tbingen for HPV testing.

After exclusion of ineligible samples a total of 1,741 specimens from women with abnormal, ASC-US and normal cytology were analyzed both by the Cervista HPV HR assay and by the Digene Hybrid Capture 2 High Risk DNA Test. This collection also included 60 samples of patients with CIN3+, which were added to the cohort in order to obtain a higher CIN3+ rate. Samples with discordant HPV test results were genotyped by INNO-LiPA HPV Genotyping Extra for discrepancy analysis. Participants were blinded for their HPV test results, and colposcopy and histopathology was only performed when indicated. Samples with a primary histology of CIN2+ were independently reviewed.

\section{Sample collection}

According to routine guidelines all clinical samples were collected in LBC PreservCyt Collection medium (Hologic) using the Cervex broom and were subsequently tested by cytology in the central cytology laboratory within one week of collection.

\section{Liquid based cytology}

All samples were tested by the ThinPrep 2000 Processor (Hologic) following the manufacturers instructions. Cytology results were reported using the Munich
Nomenclature II and were translated into The Bethesda System (TBS) (Table 1). LBC results were considered negative when the result was Pap I/II or Pap IIw; all other results were considered positive.

\section{DNA extraction and HPV testing}

Residual LBC samples were sent to the central molecular testing laboratory (UKT, Tbingen) within 2 weeks of collection for HPV testing. For testing by Cervista HPV HR assay, DNA extraction was performed on $2 \mathrm{ml}$ of each ThinPrep sample using the GenFind DNA Extraction Kit in combination with the ThinPrep 5000 STS (Sample Transfer System, (Hologic). To test for a possible risk of cross-contamination during sample transfer by the STS, we ran a pilot experiment with alternating blank samples and samples containing HPV16-positive SiHa cells and found no evidence of cross-contamination. DNA extraction was carried out according to the manufacturers instructions. DNA integrity was measured by PCR using primers targeting the human $\beta$-globin gene. Using the Cervista high throughput automation (HTA) system DNA samples (10 $\mu$ l per reaction) were then tested by Cervista HPV HR assay, which was performed in compliance with the manufacturer s instructions. The current cut-off definitions were used in this study, which were calculated as per the manufacturers instructions. Briefly, a signal-to-noise value was generated for each of three reactions, which is referred to as Fold-Over-Zero (FOZ). A FOZ ratio was calculated by dividing the highest by the lowest FOZ value of the three reactions. A given sample was considered HPVpositive if the HPV FOZ ratio was $\geq 1.525$. When the FOZ ratio was $<1.525$, but all three individual reaction FOZ values were $\geq 1.93$, the sample was also considered positive for HPV.

$4 \mathrm{ml}$ of each ThinPrep sample were processed for HR $\mathrm{HC} 2$ testing, which was performed using the Rapid Capture System 1 (RCS-1) according to the manufacturer $\mathrm{s}$ instructions. The cut-off value of RLU/CO $=1$, equivalent to

Table 1 Results LBC versus histology and HPV testing

\begin{tabular}{|c|c|c|c|c|c|c|c|}
\hline & \multirow[b]{2}{*}{ Not performed } & \multicolumn{5}{|c|}{ Primary Histology $(\mathrm{N}=139)$} & \multirow[b]{2}{*}{ Total } \\
\hline & & Normal & Borderline & CIN1 & CIN2 & CIN3+ & \\
\hline LBC & $\mathrm{N}(\mathrm{Cer}+, \mathrm{HC} 2+)$ & $\mathrm{N}(\mathrm{Cer}+, \mathrm{HC} 2+)$ & $\mathrm{N}(\mathrm{Cer}+, \mathrm{HC} 2+)$ & $\mathrm{N}(\mathrm{Cer}+, \mathrm{HC} 2+)$ & $\mathrm{N}(\mathrm{Cer}+, \mathrm{HC} 2+)$ & $\mathrm{N}(\mathrm{Cer}+, \mathrm{HC} 2+)$ & $\mathrm{N}(\mathrm{Cer}+, \mathrm{HC} 2+)$ \\
\hline Normal (Pap I/II) & $1,208(130,69)$ & 0 & 0 & 0 & 0 & 0 & $1,208(130,69)$ \\
\hline ASC-US (Pap $\| w)$ & $65(20,18)$ & 0 & 0 & 0 & 0 & 0 & $65(20,18)$ \\
\hline ASC-H, AGUS (Pap III) & $14(6,5)$ & 0 & $1(0,0)$ & 0 & 0 & $5(4,4)$ & $20(10,9)$ \\
\hline LSIL, HSIL (Pap IIID) & $312(197,211)$ & $21(16,18)$ & $1(1,1)$ & $5(4,4)$ & $13(12,13)$ & $20(20,20)$ & $372(250,267)$ \\
\hline HSIL, CIS (Pap IVa) & $3(3,3)$ & $1(1,1)$ & 0 & $3(3,3)$ & $6(6,6)$ & $58(56,58)$ & $71(69,71)$ \\
\hline HSIL, CIS, Micro (IVb) & 0 & 0 & 0 & 0 & 0 & $2(2,2)$ & $2(2,2)$ \\
\hline Microinvasive (Pap V) & 0 & 0 & 0 & 0 & 0 & $3(3,3)$ & $3(3,3)$ \\
\hline Total & $1,602(356,306)$ & $22(17,19)$ & $2(1,1)$ & $8(7,7)$ & $19(18,19)$ & $88(85,87)$ & $1,741(484,439)$ \\
\hline
\end{tabular}

Cer+: Cervista positive; $\mathrm{HC2}+\mathrm{HC2}$ positive. 
1pg HPV DNA per $1 \mathrm{ml}$ of sampling buffer for positive test results, was used in this study. PreservCyt specimens were retested when RLU/CO ratios between $\geq 1.0$ and $<2.5$ were obtained. If the initial retest result was positive (RLU/CO of $\geq 1.0$ ), the specimen was reported as positive . If the retest was negative (RLU/CO of $<1.0$ ), a second repeat test (third result) was performed to generate a final result.

HPV genotyping was carried out using the INNOLiPA HPV Genotyping Extra test as previously described [23].

\section{Histology review}

All samples with a primary histology result of CIN2+ were reviewed by an independent external expert. In case of a discrepant review reading, a second histology review was performed. If two out of three diagnoses were identical, the result was considered final.

\section{Statistical analysis}

Statistical analysis was performed on all samples with valid test results from $\mathrm{LBC}$, the Cervista HPV HR test and $\mathrm{HC} 2(\mathrm{~N}=1,741)$. Cohen s kappa value $(\mathrm{K})$ was used to calculate the agreement between the Cervista HPV $\mathrm{HR}$ test and the $\mathrm{HC} 2$ test. $95 \%$ confidence intervals (CI) for HPV prevalence were calculated using the Wilson score method. McNemars test for comparison of two proportions was used for calculating two-sided P-values to assess statistical significance of different Cervista HPV HR and HC2 test results. In addition, relative sensitivities and specificities as well as positive predictive values (PPV) and negative predictive values (NPV) based on cytological results and histology were calculated according to Cuzick et al. [24]. The statistics software package $\mathrm{R}$ version 3.0.2. was used for statistical analyses.

\section{Ratio analysis of sensitivities and specificities}

Relative performance of the tests were measured by calculating the ratio of sensitivity and the ratio of specificity. The ratio of two sensitivities is the True Positive rate of test 1 divided by the True Positive rate of test 2 . The ratio of two specificities depends on the prevalence, which in this case was $\operatorname{Spec}(\mathrm{CER}) / \mathrm{Spec}(\mathrm{HC} 2)=(0.77$ Prevalence $) /(0.80$ Prevalence). A full description of this method can be found in the Additional file 1. Confidence intervals were calculated using the delta method transform on the proportion s confidence interval. The ratio analysis has been performed using the statistics software package $\mathrm{R}$ version 3.0.2.

\section{Results}

Out of 2,303 overall specimens, a total of 562 were excluded due to inadequacy $(\mathrm{n}=196)$, insufficient material $(n=262)$ or because study participants were younger than 30 years $(\mathrm{n}=104$; Figure 1$)$. The 1,741 remaining specimens were analysed by both the Cervista HPV HR assay and HC2. 468 of these samples had an abnormal cytology result (Pap $\geq \mathrm{III}$ ), 65 were classified ASC-US (Pap IIw) and 1,208 women were cytologically normal (Pap I/II) (Table 1). Colposcopy and histopathology was performed on 139 patients with abnormal cytology results and was not performed on cytologically normal women due to the purely observational study design. A high CIN3+ rate was obtained by including 60 additional patients with confirmed CIN3+.

\section{HPV prevalence detected by the Cervista HPV HR test and $\mathrm{HC2}$}

Concordant results were obtained from 1,608 (92.4\%) of 1,741 LBC samples (Table 2). The agreement between the tests was excellent at $\mathrm{K}=0.8$ (95\% CI: 0.77-0.84). Overall HPV detection rates by the Cervista HPV HR test and $\mathrm{HC} 2$ for all $\mathrm{LBC}$ categories are summarized in Table 3. In women with normal LBC results, HR HPV was detected in $10.8 \%$ of the specimens by the Cervista HPV HR test and in $5.7 \%$ using the $\mathrm{HC} 2$ test $(\kappa=0.53$ (0.43-0.61)). HPV prevalence detected in the ASC-US category (Pap IIw) was $30.8 \%$ for the Cervista HPV HR assay and $27.7 \%$ for $\mathrm{HC} 2$, respectively. For category ASC-H and AGUS (Pap III), Cervista HPV HR testpositivity was $50 \%$ compared to $45 \%$ for HC2; for LSIL, HSIL (Pap IIID) $67.2 \%$ and $71.8 \%$ and for HSIL, CIS (Pap IVa) $97.2 \%$ and 100\%, respectively. 100\% HPVpositivity was determined for both tests in the cytology categories HSIL (Pap IIID), CIS (IVb) and microinvasive (Pap V). Interestingly, kappa values ( $\mathrm{\kappa}$ ) calculated to measure the agreement of both HPV tests were only fair $(\kappa \leq 0.55)$ for LBC categories $\leq$ ASC-US ( $\leq$ Pap IIw). For categories AGUS+ ( $\geq$ Pap III) the agreement was excellent (0.83 (0.77-0.88); Table 3).

\section{Discrepancy analysis}

A total of 133 discordant samples were detected between the Cervista HPV HR test and HC2 (Table 4). 89 discrepant samples (66.9\%) were Cervista HPV HR test-positive and HC2-negative compared to only 44 samples (31.7\%) with $\mathrm{HC} 2$-positive and Cervista-negative test results. The

Table 2 Comparison of Cervista HPV HR test and HC2 results

\begin{tabular}{lllll}
\hline & & \multicolumn{3}{c}{ HR HC2 } \\
\cline { 3 - 5 } & & $\begin{array}{l}\text { Positive } \\
\text { (N/\%) }\end{array}$ & $\begin{array}{l}\text { Negative } \\
\text { (N/\%) }\end{array}$ & $\begin{array}{l}\text { Total } \\
\text { (N/\%) }\end{array}$ \\
\hline \multirow{3}{*}{ CER } & Positive & $395 / 22.7 \%$ & $89 / 5.1 \%$ & $484 / 27.8 \%$ \\
& Negative & $44 / 2.5 \%$ & $1,213 / 69.7 \%$ & $1,257 / 72.2 \%$ \\
& Total & $439 / 25.2 \%$ & $1,302 / 74.8 \%$ & $1,741 / 100 \%$ \\
\hline
\end{tabular}

The overall agreement between Cervista (CER) and $\mathrm{HC} 2$ test results was $\mathrm{K}=0.8$ (95\% Cl: 0.77-0.84). 
Table 3 HPV prevalence detected by Cervista and HC2 in comparison to LBC

\begin{tabular}{llll}
\hline LBC & CER+ve (\%) & HR HC2+ve (\%) & K (95\% Cl) \\
\hline Normal (Pap I/II) & $10.8 \%$ & $5.7 \%$ & $0.53(0.43-0.61)$ \\
ASC-US (Pap IIw) & $30.8 \%$ & $27.7 \%$ & $0.55(0.33-0.78)$ \\
ASC-H, AGUS (Pap III) & $50.0 \%$ & $45.0 \%$ & $0.9(0.71-1.0)$ \\
LSIL, HSIL (Pap IIID) & $67.2 \%$ & $71.5 \%$ & $0.82(0.75-0.88)$ \\
HSIL, CIS (Pap IVa) & $97.2 \%$ & $100 \%$ & 1 \\
HSIL, CIS, Micro (IVb) & $100 \%$ & $100 \%$ & 1 \\
Microinvasive (Pap V) & $100 \%$ & $100 \%$ & 1 \\
AGUS+ ( $\geq$ Pap III) & $71.3 \%$ & $75.2 \%$ & $0.83(0.77-0.88)$ \\
HSIL+ ( $\geq$ Pap IVa) & $100 \%$ & $100 \%$ & 1 \\
Total & $27.8 \%$ & $25.2 \%$ & $0.8(0.77-0.84)$ \\
\hline
\end{tabular}

+ve: positive; +: and higher.

95\% Cl for HPV prevalence were calculated using the Wilson Score method

majority of discordant results $(n=89)$ was detected in specimens with normal cytology, followed by the LSIL and HSIL category. Within the Pap normal category, $84.3 \%$ of the discordant samples were CER- positive and HC2negative in contrast to the PapIIID (LSIL, HSIL) group where $79.3 \%$ of the discordant samples were positive by HC2 and negative by Cervista HPV HR-test results.

By genotyping of all deviant samples $(n=133)$ using the INNO-LiPA HPV Genotyping Extra test (Table 5), we resolved 56 HC2-negative and 3 CER-negative samples as true negatives revealing an unexpectedly high number of false positive results for Cervista $(n=56)$. While a total of 21 samples were inadequate for LiPA genotyping, all remaining samples $(n=53)$ were HPV DNA positive. In detail, 16 specimens missed by Cervista contained non-target types of the Cervista HPV HR assay (including 2 HPVX types) and a total of 23 samples $(52.3 \%)$ were false-negative by Cervista. These included 3 specimens with CIN2+ histology. In contrast, 7 discordant samples (7.9\%) with negative $\mathrm{HC} 2$ and positive Cervista HPV HR test results were false-negative by $\mathrm{HC} 2$, while another 7 samples contained non-target types of the HC2 test (including 2 HPVX types). However,

Table 4 Distribution of discordant results by $L B C$ results

\begin{tabular}{llll}
\hline LBC & $\begin{array}{l}\text { HR HC2-ve, } \\
\text { CER+ve (N/\%) }\end{array}$ & $\begin{array}{l}\text { CER-ve, HR } \\
\text { HC2+ve (N/\%) }\end{array}$ & Total (N) \\
\hline Normal (Pap I/II) & $75 / 84.3 \%$ & $14 / 15.7 \%$ & 89 \\
ASC-US (Pap IIw) & $7 / 58.3 \%$ & $5 / 41.7 \%$ & 12 \\
ASC-H, AGUS (Pap III) & $1 / 100.0 \%$ & 0 & 1 \\
LSIL, HSIL (Pap IIID) & $6 / 20.7 \%$ & $23 / 79.3 \%$ & 29 \\
HSIL, CIS (Pap IVa) & 0 & $2 / 100.0 \%$ & 2 \\
Total & 89 & 44 & 133 \\
\hline
\end{tabular}

+ve: positive; -ve: negative. none of the samples missed by $\mathrm{HC} 2$ had a histology result of CIN2+.

\section{Sensitivity and specificity}

Relative sensitivity and specificity were calculated in order to measure clinical outcomes related to HPV test results. A total of 139 biopsies were taken from patients with abnormal cytology when indicated and analysed by histopathology (Table 1). Overall relative sensitivity for detection of histologically confirmed CIN3+ in patients with abnormal cytology (AGUS+) was 96.6\% for the Cervista HPV HR test and $98.9 \%$ for the $\mathrm{HC} 2$ test (Table 6). Relative specificity and PPV for Cervista HPV HR test were 15.7\% and 66.4\% or $9.8 \%$ and $65.4 \%$ for $\mathrm{HC} 2$ test, respectively.

As only women with high grade abnormal cytology were referred to colposcopy and considering the low sensitivity of cytology in detecting CIN3+ [25], the true extent of high grade CIN in the Pap I/II and IIw categories remains unknown. This lack of data prevents the application of conventional clinical sensitivity and specificity calculations. However, by calculating the ratio of the sensitivity or specificity of two tests, we developed a method, which yields a value that measures relative performance. The ratio of sensitivity between Cervista HPV HR test and HC2 is $0.977(0.69,1.27)$. The ratio of specificity also contained 1 over all prevalence possibilities. Figure 2 shows the ratio of specificity given a range of prevalences. As a result, there is no statistical difference between clinical sensitivity and specificity of the Cervista HPV HR test and HC2 for the detection of high grade disease at the $95 \%$ confidence level.

$\mathrm{N}=100$ histology reviews of slides with a primary histology result of CIN2+ were performed in the 19 CIN2 samples and in 81 of the 88 CIN3+ samples. Due to the review 5 samples changed from CIN2 to the final result CIN3 and one sample changed from a primary histology result CIN3 to a final histology CIN2. Sensitivity and specificity results for the performance of the Cervista HPV HR test versus $\mathrm{HC} 2$ with regard to the endpoint CIN3+ did not change when the review results were accounted for in the calculations (data not shown).

\section{Different Cervista cut-off values reduce HPV-positivity without affecting detection of CIN3+}

We found a significantly higher positivity rate of the Cervista HPV HR test (10.8\% positive results for Cervista versus $5.7 \%$ for $\mathrm{HC} 2$; $\mathrm{p}<0.0001$ ) in cytologically normal women (Table 3) and discrepancy analysis revealed that 56 true-negative samples were false-positive by Cervista. Therefore, we also calculated HPV positivity as detected by the Cervista HPV HR test using two different modified cutoff criteria (Table 7), which were selected for the detection of CIN3+ lesions. The manufacturer-recommended cut-off is a Fold-Over-Zero (FOZ) ratio of $\geq 1.525$ or $<1.525$ in 
Table 5 Discordant HPV test results resolved by their HPV genotype detected by INNO-LiPA genotyping

\begin{tabular}{lllll}
\hline HPV genotype & HPV classification & HR HC2-ve, CER+ve (N) & CER-ve, HR HC2+ve (N) & Histology CIN2+ (HC2-ve/CER-ve) \\
\hline HPV16* & HR & 4 & 4 & $0 / 2$ \\
HPV18* & HR & 0 & 1 & $0 / 1$ \\
HPV31* & HR & 0 & 3 & 0 \\
HPV33* & HR & 0 & 1 & 0 \\
HPV39* & HR & 1 & 1 & 0 \\
HPV51* & HR & 1 & 6 & 0 \\
HPV52* & HR & 1 & 0 & 0 \\
HPV66** & HR & 2 & 1 & 0 \\
HPV68* & Intermediate & 0 & 2 & 0 \\
HPV53 & Intermediate & 1 & 11 & 0 \\
HPV6 & LR & 0 & 1 & 0 \\
HPV54 & LR & 0 & 1 & 0 \\
HPV69/71 & LR & 1 & 0 & 0 \\
HPV74 & LR & 1 & 1 & 0 \\
HPVX & & 2 & 2 & 0 \\
HPV DNA negative & & 56 & 3 & 0 \\
no result (sample failed) & & 89 & 2 & 3 \\
Total & & 49 & 0 \\
\hline
\end{tabular}

*HC2 and Cervista target types; ${ }^{* *}$ Cervista target type; HPVX: HPV DNA was detected by LiPA, but could not be correlated to a specific type; +ve: positive; -ve: negative.

Table 6 Relative sensitivity, relative specificity, PPV and NPV of the Cervista HPV HR test and HC2 for detecting CIN3+ within various cytology groups

\begin{tabular}{|c|c|c|c|c|c|c|c|c|}
\hline \multirow[b]{3}{*}{ CIN 3+ } & \multicolumn{8}{|c|}{ CERVISTA } \\
\hline & \multicolumn{2}{|c|}{ Relative sensitivity } & \multicolumn{2}{|c|}{ Relative specificity } & \multicolumn{2}{|l|}{ PPV } & \multicolumn{2}{|l|}{ NPV } \\
\hline & $\%$ & $95 \% \mathrm{Cl}$ & $\%$ & $95 \% \mathrm{Cl}$ & $\%$ & $95 \% \mathrm{Cl}$ & $\%$ & $95 \% \mathrm{Cl}$ \\
\hline ASC-H, AGUS (Pap III) & $80.0 \%$ & $(29.9-98.9)$ & $100.0 \%$ & $(54.6-100)$ & $100.0 \%$ & $(39.6-100)$ & $50.0 \%$ & $(26.7-97.3)$ \\
\hline LSIL, HSIL (Pap IIID) & $100.0 \%$ & $(80.0-100)$ & $17.5 \%$ & $(7.9-33.4)$ & $37.7 \%$ & $(25.1-52.1)$ & $100.0 \%$ & $(56.1-100)$ \\
\hline HSIL, CIS (Pap IVa) & $96.6 \%$ & $(87.0-99.4)$ & NA & & $84.8 \%$ & $(73.4-92.1)$ & NA & \\
\hline HSIL, CIS, Micro (IVb) & $100.0 \%$ & $(19.8-100)$ & NA & & $100 \%$ & $(19.8-100)$ & NA & \\
\hline Microinvasive (Pap V) & $100.0 \%$ & $(30.1-100)$ & NA & & $100 \%$ & $(30.1-100)$ & NA & \\
\hline AGUS+ ( $\geq$ Pap III) & $96.6 \%$ & $(89.7-99.1)$ & $15.7 \%$ & $(7.5-29.1)$ & $66.4 \%$ & $(57.5-74.4)$ & $72.7 \%$ & $(39.3-92.7)$ \\
\hline \multirow[t]{3}{*}{ HSIL+ ( $\geq$ Pap IVa) } & $96.8 \%$ & (88.0-99.4) & NA & & $85.9 \%$ & $(75.2-92.7)$ & NA & \\
\hline & \multicolumn{8}{|l|}{ HR HC2 } \\
\hline & \multicolumn{2}{|c|}{ Relative sensitivity } & \multicolumn{2}{|c|}{ Relative specificity } & \multicolumn{2}{|l|}{ PPV } & \multicolumn{2}{|l|}{ NPV } \\
\hline CIN 3+ & $\%$ & $95 \% \mathrm{Cl}$ & $\%$ & $95 \% \mathrm{Cl}$ & $\%$ & $95 \% \mathrm{Cl}$ & $\%$ & $95 \% \mathrm{Cl}$ \\
\hline ASC-H, AGUS (Pap III) & $80.0 \%$ & $(29.9-98.9)$ & $100.0 \%$ & $(54.6-100)$ & $100.0 \%$ & $(39.6-100)$ & $50.0 \%$ & $(26.7-97.3)$ \\
\hline LSIL, HSIL (Pap IIID) & $100.0 \%$ & $(80.0-100)$ & $10.0 \%$ & $(3.3-24.6)$ & $35.7 \%$ & $(23.7-49.7)$ & $100.0 \%$ & $(39.6-100)$ \\
\hline HSIL, CIS (Pap IVa) & $100.0 \%$ & $(92.3-100)$ & NA & & $85.3 \%$ & $(74.2-92.3)$ & NA & \\
\hline HSIL, CIS, Micro (IVb) & $100.0 \%$ & $(19.8-100)$ & NA & & $100 \%$ & $(19.8-100)$ & NA & \\
\hline Microinvasive (Pap V) & $100.0 \%$ & $(30.1-100)$ & NA & & $100 \%$ & $(30.1-100)$ & NA & \\
\hline AGUS+ ( $\geq$ Pap III) & $98.9 \%$ & $(92.9-99.9)$ & $9.8 \%$ & $(3.7-22.2)$ & $65.4 \%$ & $(56.6-73.3)$ & $83.3 \%$ & $(36.5-99.1)$ \\
\hline HSIL+ ( $\geq$ Pap IVa) & $100.0 \%$ & $(92.8-100)$ & NA & & $86.3 \%$ & $(75.8-92.9)$ & NA & \\
\hline
\end{tabular}

+: and higher. 


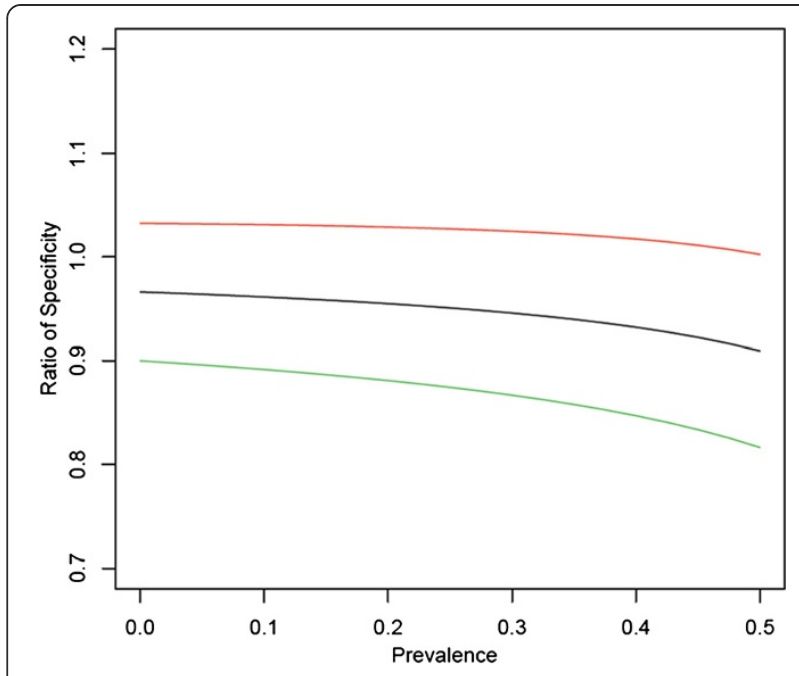

Figure 2 Ratio of specificities of Cervista and HC2 as a function of HR HPV prevalence.

combination with an individual-reaction $\mathrm{FOZ}$ value of $>1.93$ if all three probe sets yielded a positive test result. We here examined the cut-off FOZ ratio $\geq 1.525$ without any additional criteria and a FOZ ratio of $\geq 1.525$ or $<1.525$ with an individual-reaction FOZ value of $\geq 4.0$. As shown in Table 7, HPV prevalence measured by the Cervista HPV HR test in the LBC normal category decreased to $6.7 \%$ and $6.1 \%$, respectively, whereas the kappa values increased to 0.73 when using the two different FOZ cut-off values. Relative sensitivity for the detection of CIN3+ was not affected by the modification of the FOZ-values.

\section{Discussion}

This study compares the Cervista HPV HR test to the $\mathrm{HC} 2$ test within a German routine cervical cancer screening population of women aged $\geq 30$ years initially screened by LBC. Overall the HR HPV prevalence was $27.8 \%$ and $25.2 \%$ for Cervista HPV HR and HC2, respectively. Due to the inclusion of 60 additional patients with $\mathrm{CIN} 3+$, the overall prevalence is higher than HR HPV prevalence rates of $\sim 9 \%$ previously reported for a comparable screening population with participants aged $\geq 30$ years [14]. The overall agreement of the HPV-tests was excellent $(\kappa=0.8)$. Relative performance of each HPV test was assessed by calculating relative sensitivity and specificity based on histologically confirmed CIN3+ as positive clinical outcome. The relative sensitivities determined for both tests in women with abnormal cytology (AGUS+) were comparable, with $96.6 \%$ for Cervista and 98.9\% for HC2. Although relative specificity was better for the Cervista HPV HR test (15.7\%) than for HC2 (9.8\%), the difference was not significant ( $\mathrm{p}$ value $=0.14$ ), which is supported by the results obtained from our ratio analysis. Our findings confirm the results of previous publications, which compared the performances of the Cervista HPV HR test and the HC2 tests and showed no significant differences in sensitivities detecting histologically confirmed high grade lesions (CIN2+ or CIN3+) [12,16,17]. These studies also demonstrated differences in specificities in favour for the Cervista HPV HR test, however only Belinson et al. was able to show that this difference was of statistical significance [17].

Interestingly, we found a significant difference between HR HPV prevalences detected by Cervista and HC2 in the cytology normal (NILM; negative for intraepithelial lesion or malignancy) group ( $<0.0001$; Table 3$)$. In order to resolve discordant results genotyping by INNO-LiPA Extra was performed. LiPA genotyping has previously been used as an adjudicating assay in test comparison studies [26,27] because of its excellent analytical sensitivity of 20 to 70 copies per assay [28]. In line with previous studies (summarized by [29]) our discrepancy analysis demonstrated substantial cross-reactivity of $\mathrm{HC} 2$ with non-target HPV types ( $n=16$ versus $n=3$ for HC2 and Cervista, respectively). Furthermore, discrepancy analysis revealed a high false positive rate for Cervista using the currently approved cut-off values $(\mathrm{n}=56$ for Cervista versus $\mathrm{n}=3$ for $\mathrm{HC2}$; Table 5). This result, however, is not in line with previous studies demonstrating a tendency of a lower or no difference in the HR HPV prevalence rate for Cervista in comparison to $\mathrm{HC} 2$ in women with NILM cytology [12-17]. This disagreement with previous reports might, however reflect differences in study design $[12,13,16,17]$, age range of participants [12,13,15-17] or overall HR HPV prevalence rates [14]. Nonetheless, our data confirm a commentary, which critically discusses the high false positive detection rate of the Cervista HPV HR test in cytologically normal specimens of women $\geq 30$ years of age by evaluating data published with the Cervista HPV HR test package insert [30]. By applying modified cut-off values

Table 7 Different cut-off values for Cervista HPV HR test in LBC-normal samples

\begin{tabular}{lllll}
\hline CERVISTA cut-off & & & & HC2+ve \% \\
\hline FOZ ratio & FOZ value & CER+ve $\%$ & K (95\% Cl) \\
\hline$\geq 1.525$ or $<1.525$ & $\geq 1.93$ & $10.8 \%$ & $5.7 \%$ & $0.52(0.42-0.61)$ \\
$\geq 1.525$ & & $6.7 \%$ & $5.7 \%$ & $0.73(0.64-0.81)$ \\
$\geq 1.525$ or $<1.525$ & $\geq 4$ & $6.1 \%$ & $5.7 \%$ & $0.73(0.65-0.81)$ \\
\hline
\end{tabular}

+ve: positive. 
(increase of the individual-reaction FOZ value from $\geq 1.93$ to $\geq 4$ or the elimination of the individual-reaction FOZ value), which were optimised for the detection of CIN3+ lesions and not HPV DNA in cytologically normal samples, we were able to dramatically reduce the false-positive rate of the Cervista HPV HR test in the Pap normal group resulting in a similar HR HPV-prevalence as detected by $\mathrm{HC} 2$, while the relative sensitivity for the detection of high grade disease (CIN3+) remained unaltered. Therefore, a re-adjustment of the manufacturer s cut-off values for the use of the Cervista HPV HR test in primary screening may be considered, but further population-based studies are necessary to confirm our finding. Studies evaluating the company set cut-off values for the detection of disease have previously been performed for other HPV tests including $\mathrm{HC} 2$ where adjustment led to an increased specificity of disease detection and fewer false-positive test results [31]. It is worth noting that current clinical routine in Germany requires ancillary HPV testing only for patients with abnormal or borderline cytology results (PapIIw, PapIII and PapIIID). Importantly, as around 50\% of ASC-US specimens will be tested HR HPV positive [32], the accurate early detection of relevant infections by non-invasive and cost-effective tests is fundamental. For the ASC-US category, we demonstrate that the performance of the Cervista HPV HR test was non-inferior to $\mathrm{HC} 2$ and rather showed a higher detection rate than $\mathrm{HC} 2$. This is in line with previous studies demonstrating a high sensitivity for HR HPV detection and a lower false positive rate as compared to $\mathrm{HC} 2$ in ASC-US specimens $[13,14,18,19,21]$. Finally, split sample test comparison results of multiple HPV tests other than Cervista have recently been published from the Horizon study where the authors found substantial disagreement between HPV tests especially in women aged 3065 years. These disagreements were attributed to screening false positives and were probably based on the different assay designs [33].

\section{Conclusions}

We evaluated the performance of the Cervista HPV HR test using residual $\mathrm{LBC}$ specimens of a German routine cervical cancer screening population. We demonstrate that the Cervista HPV HR test would be appropriate to be used in primary routine cervical cancer screening after cut-off adjustment or in its current format adjunctive to cytology for detection of high grade cervical intraepithelial neoplasia in women with abnormal (ASCUS+) cytology, yielding similar HR HPV detection rates and relative sensitivity and specificity as the $\mathrm{HC} 2$ test. The presence of an internal control as provided by the Cervista HPV HR assay might be responsible for the reduced false negative test results within discrepant samples, as compared to the $\mathrm{HC} 2$ test system, which lacks such a control. Another major advantage of the Cervista
HPV HR test is the automated integrated DNA extraction step using the HTA system from Hologic, which avoids extra working steps resulting in a minimised risk of sample contamination prior to testing.

\section{Additional file}

\section{Additional file 1: Supplemental methods.}

\section{Competing interests}

This study was supported by an unconditional grant from Hologic Incorporated to the University Hospital of Tbingen, which hosts the Division of Experimental Virology. Hologic had no influence on the study protocol, no access to primary data and was not involved in the analysis and writing of the manuscript.

\section{Authors contributions}

GB recruited study participants, collected the samples and performed LBC. Al performed HPV testing and genotyping. RvW performed histologies. LW and PM performed the Ratio of specificities and sensitivities analysis and were involved in drafting the manuscript. $\mathrm{BH}$ was the studies coordinator and performed basic statistical analyses. JH performed statistical analyses and drafted the manuscript. TI designed the study, was involved in data analysis and interpretation and drafted the manuscript. All authors read and approved the final manuscript.

\section{Acknowledgements}

This study was funded by an unconditional grant from Hologic Inc. Furthermore, publication of this manuscript was supported by the Deutsche Forschungsgemeinschaft and the Open Access Publishing Fund of the Tbingen University.

\section{Author details}

${ }^{1}$ German Clinic Bad Mnder, Bad Mnder, Germany. $\quad{ }^{2}$ Clinical Epidemiology and Applied Biometry, University Hospital Tbingen, Tbingen, Germany. ${ }^{3}$ Division of Experimental Virology, Institute of Medical Virology, University Hospital Tbingen, Elfriede-Aulhorn-Str. 6, 72076 Tbingen, Germany.

Received: 15 May 2014 Accepted: 1 December 2014

Published online: 09 December 2014

\section{References}

1. Schenck U, von Karsa L: Cervical cancer screening in Germany. Eur J Cancer 2000, 36(17):2221 2226

2. Krebs in Deutschland 2007/2008. Robert Koch-Institut und die Gesellschaft der epidemiologischen Krebsregister in Deutschland eV, Berlin 2012, 8. Ausgabe.

3. Siebert U, Sroczynski G, Hillemanns P, Engel J, Stabenow R, Stegmaier C, Voigt K, Gibis B, Holzel D, Goldie SJ: The German cervical cancer screening model: development and validation of a decision-analytic model for cervical cancer screening in Germany. Eur J Pub Health 2006, 16(2):185 192.

4. Bosch FX, Lorincz A, Munoz N, Meijer CJ, Shah KV: The causal relation between human papillomavirus and cervical cancer. J Clin Pathol 2002, 55(4):244 265

5. Schiffman M, Clifford G, Buonaguro FM: Classification of weakly carcinogenic human papillomavirus types: addressing the limits of epidemiology at the borderline. Infect Agents Cancer 2009, 4:8.

6. Munoz N, Bosch FX, de Sanjose S, Herrero R, Castellsague X, Shah KV, Snijders PJ, Meijer CJ, International Agency for Research on Cancer Multicenter Cervical Cancer Study G: Epidemiologic classification of human papillomavirus types associated with cervical cancer. $N$ Engl J Med 2003, 348(6):518 527.

7. Arbyn M, Ronco G, Anttila A, Meijer CJ, Poljak M, Ogilvie G, Koliopoulos G, Naucler P, Sankaranarayanan R, Peto J: Evidence regarding human papillomavirus testing in secondary prevention of cervical cancer. Vaccine 2012, 30(Suppl 5):F88 F99.

8. Dillner J: Primary human papillomavirus testing in organized cervical screening. Curr Opin Obstet Gyn 2013, 25(1):11 16. 
9. Castle PE, de Sanjose S, Qiao YL, Belinson JL, Lazcano-Ponce E, Kinney W: Introduction of human papillomavirus DNA screening in the world: 15 years of experience. Vaccine 2012, 30(Suppl 5):F117 F122.

10. Iftner T, Villa $\amalg$ : Chapter 12: Human papillomavirus technologies. J Natl Cancer Inst Monogr 2003, 31:80 88.

11. Halec G, Alemany L, Lloveras B, Schmitt M, Alejo M, Bosch FX, Tous S, Klaustermeier JE, Guimera N, Grabe N, Lahrmann B, Gissmann L, Quint W, Bosch FX, de Sanjose S, Pawlita M, Retrospective International Survey, HPV Time Trends Study Group: Pathogenic role of the eight probably/possibly carcinogenic HPV types 26, 53, 66, 67, 68, 70, 73 and 82 in cervical cancer. J Pathol 2014, 234(4):441 451.

12. Tao K, Yang J, Yang H, Guo ZH, Hu YM, Tan ZY, Zhang F, Duan J Comparative study of the cervista and hybrid capture 2 methods in detecting high-risk human papillomavirus in cervical lesions. Diagn Cytopathol 2014, 42(3):213 217

13. Du Chateau BK, Schroeder ER, Munson E: Clinical laboratory experience with cervista HPV HR as a function of cytological classification: comparison with retrospective digene $\mathrm{HC} 2$ high-risk HPV DNA test data. J Clin Microbiol 2013, 51(3):1057 1058.

14. Quigley NB, Potter NT, Chivukula M, Knight MZ, Welch JR, Olson MC: Rate of detection of high-risk HPV with two assays in women $>/=30$ years of age. J Clin Virol: the official publication of the Pan American Society for Clinical Virology 2011, 52(1):23 27.

15. Munson E, Du Chateau BK, Bellerose B, Czarnecka J, Griep J: Clinical laboratory evaluation of Invader(R) chemistry and hybrid capture for detection of high-risk human papillomavirus in liquid-based cytology specimens. Diagn Microbiol Infect Dis 2011, 71(3):230 235.

16. Kurian EM, Caporelli ML, Baker S, Woda B, Cosar EF, Hutchinson L: Cervista HR and HPV 16/18 assays vs hybrid capture 2 assay: outcome comparison in women with negative cervical cytology. Am J Clin Pathol 2011, 136(5):808 816.

17. Belinson JL, Wu R, Belinson SE, Qu X, Yang B, Du H, Wu R, Wang C, Zhang L, Zhou Y, Liu Y, Pretorius RG: A population-based clinical trial comparing endocervical high-risk HPV testing using hybrid capture 2 and Cervista from the SHENCCAST II Study. Am J Clin Pathol 2011, 135(5):790 795.

18. Wong AK, Chan RC, Nichols WS, Bose S: Human papillomavirus (HPV) in atypical squamous cervical cytology: the Invader HPV test as a new screening assay. J Clin Microbiol 2008, 46(3):869 875.

19. Johnson LR, Starkey CR, Palmer J, Taylor J, Stout S, Holt S, Hendren R, Bock B, Waibel E, Tyree G, Miller GC: A comparison of two methods to determine the presence of high-risk HPV cervical infections. Am J Clin Pathol 2008, 130(3):401 408.

20. Ginocchio CC, Barth D, Zhang F: Comparison of the Third Wave Invader human papillomavirus (HPV) assay and the digene HPV hybrid capture 2 assay for detection of high-risk HPV DNA. J Clin Microbiol 2008, 46(5):1641 1646

21. Schutzbank TE, Jarvis C, Kahmann N, Lopez K, Weimer M, Yount A Detection of high-risk papillomavinus DNA with commercial invadertechnology-based analyte-specific reagents following automated extraction of DNA from cervical brushings in ThinPrep media. J Clin Microbiol 2007, 45(12):4067 4069.

22. Youens KE, Hosler GA, Washington PJ, Jenevein EP, Murphy KM: Clinical experience with the Cervista HPV HR assay: correlation of cytology and HPV status from 56,501 specimens. J Mol Diagn: JMD 2011, 13(2):160 166

23. Petry KU, Luyten A, Justus A, Iftner A, Strehlke S, Schulze-Rath R, Iftner T: Prevalence of low-risk HPV types and genital warts in women born $1988 / 89$ or 1983/84 -results of WOLVES, a population-based epidemiological study in Wolfsburg, Germany. BMC Infect Dis 2012, 12:367.

24. Cuzick J, Cadman L, Mesher D, Austin J, Ashdown-Barr L, Ho L, Terry G, Liddle S, Wright $C$, Lyons D, Szarewski A: Comparing the performance of six human papillomavirus tests in a screening population. $\mathrm{Br} J$ Cancer 2013, 108(4):908 913.

25. McCrory DC, Matchar DB, Bastian L, Datta S, Hasselblad V, Hickey J, Myers E, Nanda K. Evaluation of cervical cytology. Evid Rep Technol Assess 1999, 5:1 6.

26. Ngou J, Magooa MP, Gilham C, Djigma F, Didelot MN, Kelly H, Yonli A, Sawadogo B, Lewis DA, Delany-Moretlwe S, Mayaud P, Segondy M, Harp Study Group: Comparison of careHPV and hybrid capture 2 assays for detection of high-risk human Papillomavinus DNA in cervical samples from HIV-1-infected African women. J Cin Mirobiol 2013, 51(12):4240 4242.

27. Hwang $Y$, Lee $M$ : Comparison of the AdvanSure human papillomavirus screening real-time PCR, the Abbott RealTime High Risk human papillomavinus test, and the Hybrid Capture human papillomavinus DNA test for the detection of human papillomavirus. Ann Lab Med 2012, 32(3):201 205

28. Torres M, Fraile L, Echevarria J, Hernandez Novoa B, Ortiz M: Human Papillomavirus (HPV) Genotyping: Automation and Application in Routine Laboratory Testing. Open Virol J 2012, 6:144 150.

29. Gillio-Tos A, De Marco L, Carozzi FM, Del Mistro A, Girlando S, Burroni E, Frayle-Salamanca H, Giorgi Rossi P, Pierotti P, Ronco G, New Technologies for Cervical Cancer Screening (NTCC) Working Group: Clinical impact of the analytical specificity of the hybrid capture 2 test data from the New Technologies for Cervical Cancer (NTCC) study. J Clin Microbiol 2013, 51(9):2901 2907.

30. Kinney W, Stoler MH, Castle PE: Special commentary: patient safety and the next generation of HPV DNA tests. Am J Clin Pathol 2010, 134(2):193 199.

31. Rebolj M, Bonde J, Njor SH, Lynge E Human papillomavirus testing in primary cervical screening and the cut-off level for hybrid capture 2 tests: systematic review. BMJ 2011, 342:d2757.

32. Evans MF, Adamson CS, Papillo JL, St John TL, Leiman G, Cooper K: Distribution of human papillomavirus types in ThinPrep Papanicolaou tests classified according to the Bethesda 2001 teminology and correlations with patient age and biopsy outcomes. Cancer 2006, 106(5):1054 1064.

33. Rebolj M, Preisler S, Ejegod DM, Rygaard C, Lynge E, Bonde J: Disagreement between human papillomavirus assays: an unexpected challenge for the choice of an assay in primary cervical screening. PLoS One 2014, 9(1):e86835.

doi:10.1186/s12879-014-0674-

Cite this article as: Boehmer et al.: A population-based observational study comparing Cervista and Hybrid Capture 2 methods: improved relative specificity of the Cervista assay by increasing its cut-off. BMC Infectious Diseases 2014 14:674.

\section{Submit your next manuscript to BioMed Central and take full advantage of:}

$凶$ Convenient online submission

$\otimes$ Thorough peer review

凶No space constraints or color $₫$ gure charges

$\otimes$ Immediate publication on acceptance

ه Inclusion in PubMed, CAS, Scopus and Google Scholar

$\bigotimes$ Research which is freely available for redistribution

Submit your manuscript at www.biomedcentral.com/submit
C) Biomed Central 